\title{
The TAIGA experiment - a hybrid detector for very high energy gamma-ray astronomy and cosmic ray physics in the Tunka valley
}

\author{
N. Budnev ${ }^{* 2}$, I. Astapov ${ }^{9}$, P. Bezyazeekov ${ }^{2}$, V. Boreyko ${ }^{10}$, A. Borodin ${ }^{10}$, \\ M. Brueckner ${ }^{8}$, A. Chiavassa ${ }^{4}$, A. Dyachok ${ }^{2}$, O. Fedorov ${ }^{2}$, A. Gafarov ${ }^{2}$, \\ N. Gorbunov ${ }^{10}$, V. Grebenyuk ${ }^{10}$, O. Gress ${ }^{2}$, T. Gress ${ }^{2}$, O. Grishin ${ }^{2}$, A. Grinyuk ${ }^{10}$, \\ A. Haungs ${ }^{14}$, D. Horns ${ }^{6}$, T. Huege ${ }^{14}$, A. Ivanova ${ }^{2}$, N. Kalmykov ${ }^{1}$, Y. Kazarina ${ }^{2}$, \\ V. Kindin ${ }^{9}$, S. Kiryuhin ${ }^{2}$, R. Kokoulin ${ }^{9}$, K. Kompaniets ${ }^{9}$, E. Korosteleva ${ }^{1}$, \\ D. Kostunin ${ }^{14}$, V. Kozhin ${ }^{1}$, E. Kravchenko ${ }^{11,12}$, M. Kunnas ${ }^{6}$, L. Kuzmichev ${ }^{1,2}$, \\ Yu. Lemeshev ${ }^{2}$, V. Lenok ${ }^{2}$, B. Lubsandorzhiev ${ }^{1,3}$, N. Lubsandorzhiev ${ }^{1}$, R. Mirgazov ${ }^{2}$, \\ R. Mirzoyan ${ }^{5,2}$, R. Monkhoev ${ }^{2}$, R. Nachtigall ${ }^{6}$, E. Osipova ${ }^{2}$, A. Pakhorukov ${ }^{2}$, \\ M. Panasyuk ${ }^{1}$, L. Pankov ${ }^{2}$, A. Petrukhin ${ }^{9}$, V. Poleschuk ${ }^{2}$, E. Popescu ${ }^{13}$, E. Popova ${ }^{1}$, \\ A. Porelli ${ }^{8}$, E. Postnikov1, V. Prosin' ${ }^{1}$, V. Ptuskin ${ }^{7}$, E. Rjabov ${ }^{2}$, G. Rubtsov ${ }^{3}$, \\ A. Pushnin ${ }^{2}$, Y. Sagan ${ }^{10}$, B. Sabirov ${ }^{10}$, V. Samoliga ${ }^{2}$, F. Schröder ${ }^{14}$, Yu. Semeney ${ }^{2}$, \\ A. Silaev ${ }^{1}$, A. Silaev(junior) ${ }^{1}$, A. Sidorenkov ${ }^{3}$, A. Skurikhin ${ }^{1}$, V. Slunecka ${ }^{10}$, \\ A. Sokolov ${ }^{11,12}$, C. Spiering ${ }^{8}$, L. Sveshnikova ${ }^{1}$, V. Tabolenko ${ }^{2}$, B. Tarashansky ${ }^{2}$, \\ A. Tkachenko ${ }^{10}$, L. Tkachev ${ }^{10}$, M. Tluczykont $^{6}$, R. Wischnewski ${ }^{8}$, A. Zagorodnikov $^{2}$, \\ D. Zhurov ${ }^{2}$, V. Zurbanov' ${ }^{2}$, I. Yashin ${ }^{9}$, \\ ${ }^{1}$ Skobeltsyn Institute of Nuclear Physics MSU, Moscow, Russia \\ ${ }^{2}$ Institute of Applied Physics ISU, Irkutsk, Russia \\ ${ }^{3}$ Institute for Nuclear Research of RAN, Moscow, Russia \\ ${ }^{4}$ Dipartimento di Fisica Generale Universiteta di Torino and INFN, Torino, Italy \\ ${ }^{5}$ Max-Planck-Institute for Physics, Munich, Germany \\ ${ }^{6}$ Institut für Experimentalphysik, University of Hamburg, Germany \\ ${ }^{7}$ IZMIRAN, Moscow, Russia \\ ${ }^{8}$ DESY, Zeuthen, Germany \\ ${ }^{9}$ National Research Nuclear University MEPhI (Moscow Engineering Physics Institute), \\ Moscow, Russia \\ ${ }^{10}$ JINR, Dubna, Russia \\ ${ }^{11}$ Novosibirsk State University, NSU, Novosibirsk, Russia \\ ${ }^{12}$ Budker Institute of Nuclear Physics SB RAS, Novosibirsk, Russia \\ ${ }^{13}$ ISS, Bucharest, Romania \\ ${ }^{14}$ Institut für Kernphysik, Karlsruhe Institute of Technology (KIT), Germany \\ E-mail: nbudnev@api.isu.ru
}


The physics motivations and characteristics of the new gamma-experiment TAIGA (Tunka Advanced Instrument for cosmic ray physics and Gamma Astronomy) are presented. The TAIGA experiment addresses ground-based gamma-ray astronomy at energies from a few $\mathrm{TeV}$ to several $\mathrm{PeV}$, as well as cosmic ray physics from $100 \mathrm{TeV}$ to several EeV. For the energy range 30 - 200 $\mathrm{TeV}$ the sensitivity of the planned for future extension of TAIGA up to $5 \mathrm{~km}^{2}$ area for detection of the local sources is expected to be $10^{-13} \mathrm{erg} \mathrm{cm}^{-2} \mathrm{sec}^{-1}$ for $500 \mathrm{~h}$ of observation. The combination of the wide angle Cherenkov timing detectors of the TAIGA-HiSCORE array and the $4 \mathrm{~m}$ class Imaging Atmospheric Cherenkov Telescopes of the TAIGA-IACT array with their FoV of $10 \times 10$ degrees offers a cost effective-way to construct a $5 \mathrm{~km}^{2}$ array. Reconstruction of an EAS energy, direction and core position based on the TAIGA-HiSCORE data will allow us to increase the distance between comparatively expensive IACTs up to $800-1200 \mathrm{~m}$. The low investments together with the high sensitivity for energies $\geqslant 30-50 \mathrm{TeV}$ make this pioneering technique very attractive for exploring the galactic PeVatrons and cosmic rays. In addition to the Cherenkov light detectors we intend to deploy an array of muon detectors (TAIGA-Muon array) over an area of $1 \mathrm{~km}^{2}$ with a total area of about $2000-3000 \mathrm{~m}^{2}$. The TAIGA-IACT-HiSCORE together with the TAIGA-Muon array will be used for selection of gamma-ray induced EAS. At present the first stage of TAIGA have been constructed in the Tunka valley, $\sim 50 \mathrm{~km}$ West from the Lake Baikal in Siberia. Now it consists of 28 TAIGA-HiSCORE Cherenkov stations distributed over an area of $0.25 \mathrm{~km}^{2}$ and the first IACT of the TAIGA-IACT array. We are planning to test operation of the single telescope in coincidence with the HiSCORE up to shower impact distances of $\sim 500-600 \mathrm{~m}$; for this purpose we already started construction of the second imaging telescope at $300 \mathrm{~m}$ distance from the first one. During 2017 - 2019 years we intend to increase the number of the TAIGA-HiSCORE stations up to $100-120$, spread on the area of $1 \mathrm{~km}^{2}$ and to deploy 2 additional IACTs.

35th International Cosmic Ray Conference - ICRC2017

10-20 July, 2017

Bexco, Busan, Korea

* Speaker. 


\section{Introduction}

Progress of understanding of the nature of sources of high-energy cosmic rays in our Galaxy and in the Metagalaxy is driven by experiments measuring 3 type of particles: charged cosmic rays, gamma-quanta and neutrinos.

In the past, the Tunka Astrophysical Center in the Tunka Valley (50 km from Lake Baikal) was solely devoted to the charged cosmic rays. Three arrays to study charged CRs are in operation at the Tunka site: Tunka-133 [1], Tunka-Rex [2] and Tunka-Grande [3] (Fig. 1). Their measurement of the energy spectrum and mass composition is important in order to understand the acceleration limit of the Galactic CR sources and the transition from Galactic to extragalactic CR.

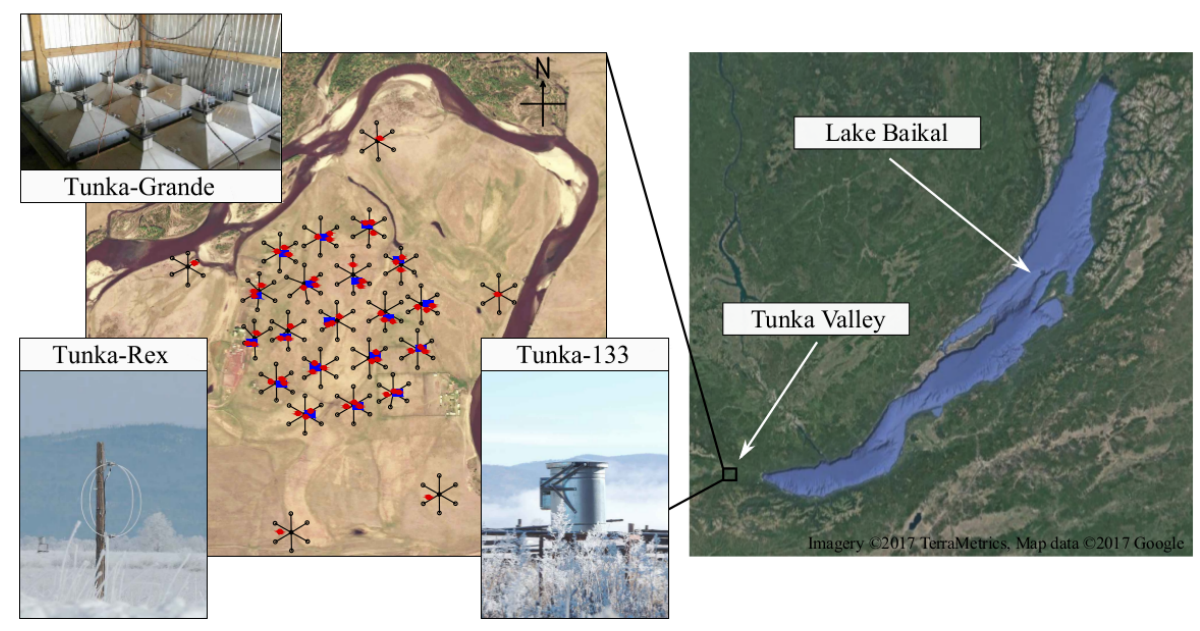

Figure 1: The EAS arrays for studying cosmic rays in Tunka Valley.

New gamma ray experiment TAIGA is designed to study gamma-radiation and charged cosmic rays in the energy range of $10^{13} \mathrm{eV}-10^{18} \mathrm{eV}$. For this energy range, there are many fundamental questions, which need to be answered. The first and foremost one is the question of the sources of Galactic cosmic rays with energies of about few $\mathrm{PeV}$, the most likely limit of the acceleration of protons in the galactic accelerators. The energy of gamma rays originated in such accelerators could extend up to $300 \mathrm{TeV}$, however so far there was no detection of a single photon with energy greater than $100 \mathrm{TeV}$.

The flux of gamma-rays strongly decreases with increasing energy and therefore it is necessary to increase the sensitive area of an array to move towards higher energies. Up to now, the vast majority of the data of gamma-ray astronomy in the $\mathrm{TeV}$ and sub- $\mathrm{TeV}$ energy range has been obtained using Imaging Atmospheric Cherenkov Telescopes (IACT), in particular with stereo systems of several such telescopes. In such approach, to build an array with large sensitive area it needs a very large number of Cherenkov telescopes. It is anticipated that the CTA project [4] will accommodate up to 70 small size telescopes (SST) over an area of $7 \mathrm{~km}^{2}$.

The TAIGA experiment [5], [6] will combine several IACTs of the TAIGA-IACT array with an array of comparatively inexpensive wide-angle non-imaging optical detectors of the TAIGAHiSCORE array [7]. This will allow us one to extend the area of the array up to several square kilometers and to considerably suppress the background from charged cosmic rays due to the su- 
perior angular resolution of HiSCORE ( $0.1^{\circ}$ at energies above $\left.100 \mathrm{TeV}\right)$. The combination of two complementary methods of the gamma-ray selection is a very effective way to construct an array with large area. There are a lot of good reasons to include the particle detectors into the hybrid detector complex of the gamma-experiment TAIGA.

Our immediate goal is to construct till the end of 2019 the first stage of TAIGA array which will consist of: $100-120$ stations distributed on an area about $1 \mathrm{~km}^{2}$, three IACTs and $200 \mathrm{~m}^{2}$ of muon detectors (Fig. 2). This setup should allow us to demonstrate the advantages of hybrid approach for high energy gamma-ray detection [8] and to start with systematic observational program.

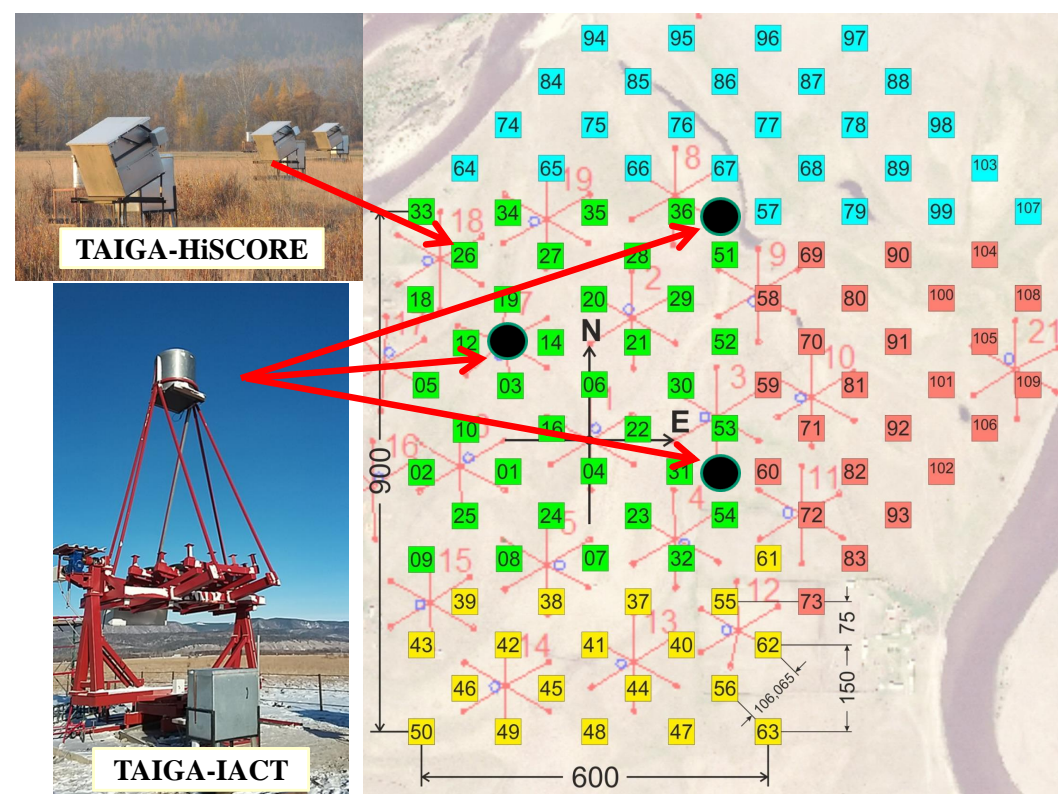

Figure 2: Planned configuration of TAIGA for 2018. Green squares - stations of the TAIGA-HiSCORE array in operation since 2015, the yellow ones - will be put into operation in 2017, the blue ones - in 2018, the red ones - in 2019. Circles - Imaging Atmospheric Cherenkov Telescopes.

\section{TAIGA-HiSCORE}

The TAIGA-HiSCORE (High Sensitivity Cosmic Origin Explorer) array is composed currently of 28 optical stations distributed in a regular grid over a surface area of $0.25 \mathrm{~km}^{2}$ with an inter-station spacing of $106 \mathrm{~m}$. All stations are tilted into the southern direction by $25^{\circ}$ to increase the time for the search of high energy gamma rays from the first test object - the Crab Nebula. For the new ones it will be possible to remotely change the zenith angle from 0 to 25 degrees. Each station contains four large area PMTs of 20 or $25 \mathrm{~cm}$ diameter, namely EMI ET9352KB, or Hamamatsu R5912 or R7081. Each PMT has a Winston cone (made of ten segments of ALANOD 4300UP foil with reflectivity $80 \%$ ), resulting in $0.5 \mathrm{~m}^{2}$ net light collection area and a field of view is of $0.6 \mathrm{sr}$ (a $30^{\circ}$ viewing half-angle).

Each station is connected with the DAQ center by a fiber-optic cable for the data transfer and synchronization. The synchronization stability of the stations reaches about $0.2 \mathrm{~ns}$, and is maintained by two independent systems (custom $100 \mathrm{MHz}$ system and White-Rabbit), see Fig.3 [10]. 
Precise calibration is achieved by using dedicated light sources. The stations operate independently, and the condition for a local (station) trigger is an excess of the summed anode pulses above the threshold of $\sim 200$ p.e., which corresponds to a Cherenkov light flux of 0.3 photon $\mathrm{cm}^{2}$ [9]. Signals from the anode and an intermediate dynode of PMTs are digitized with $0.5 \mathrm{~ns}$ sampling by a DAQboard based on the DRS-4 chip. The TAIGA-HiSCORE DAQ is presented on Fig.(3). A more detailed description of DAQ and synchronization systems is given in [10]. The new stations will be grouped in TAIGA-HiSCORE clusters with it's DAQ center. The stations included in different clusters are marked with different colors on Fig. 2.
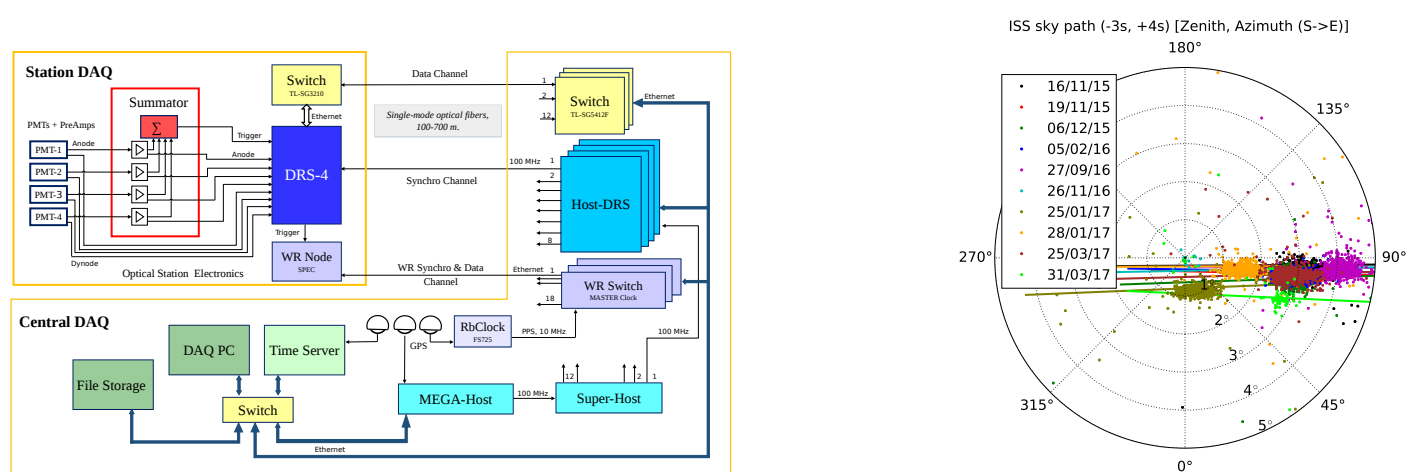

Figure 3: Left: HiSCORE Data acquisition system: stations and central part, including redundant GPS/RbClocks. Right: HiSCORE detection of the ISS-LIDAR - a summary of ten detections in 2015-2017 in the local skymap (lines: ISS track, dots: HiSCORE pointing) [16].

The reconstruction of the shower parameters is performed by using the algorithms developed for the Tunka-133 [1], [11] and for the TAIGA-HiSCORE arrays [12]. The accuracy of the reconstruction procedure was checked by comparing of the MC simulation results with experimental data [13]. About 20 millions EAS with simultaneous hits of 4 or more stations were recorded during the 55 clean moonless nights of the 2016-2017 winter season. The total time of operation was 340 hours. Main HiSCORE results are the energy spectrum of cosmic rays, a search for an event excess from the Crab Nebula and the first detection of the CATS-LIDAR onboard the ISS [14], used for precision pointing verification, see 3 . For details see [13], [15], [16].

\section{TAIGA-IACT}

In December 2016y the first IACT of the TAIGA-IACT array (Fig. 4, A) was put into test operation. The telescope is of Davis-Cotton type with 34 mirrors, $60 \mathrm{~cm}$ diameter each, with the focal length of $4.75 \mathrm{~m}$. The camera of the telescope includes 547 PMTs of XP1911 type with 19 $\mathrm{mm}$ diameter. The FOV of the camera is $9.6^{\circ} \times 9.6^{\circ}$. The camera is based on identical clusters, each including 28 PMTs (Fig. 4, B). Each cluster icludes an electronic board MAROC, the basis element of which is a 64-channel ASIC MAROC-3. A more detailed description of the camera is presented in [17].

The telescope drive system includes a motor controller and two stepper motors for axis control. For the pointing calibration of the telescope a CCD-camera is installed on the reflector dish of the telescope. The position of the CCD-camera allows us capturing both the Cherenkov camera and 

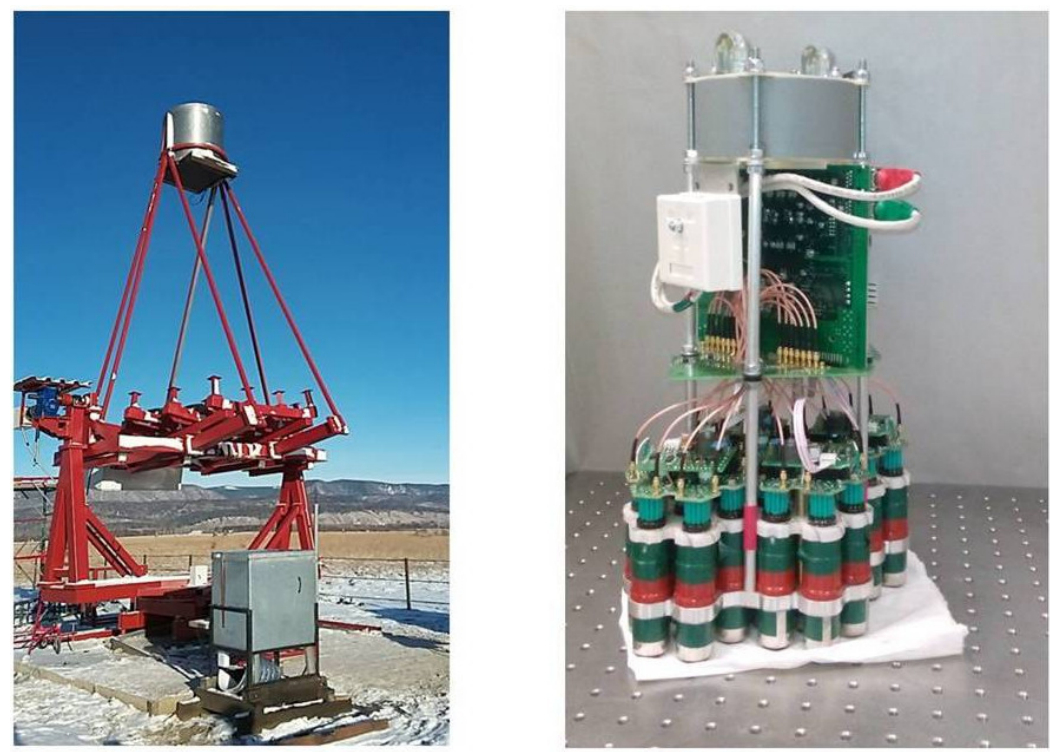

Figure 4: A: First TAIGA-IACT. B: Cluster of 28 PMTs

the observed target [18]. Test operation of the telescope was started at the end of January [19]. The trigger rate of the telescope is set to about $30 \mathrm{~Hz}$, while the counting rate of EAS events is about $2 \mathrm{~Hz}$ and the counting rate of joint events (TAIGA- IACT + HiSCORE) is about $0.3 \mathrm{~Hz}$. Figure 5 shows two examples of the EAS images with the joint trigger.
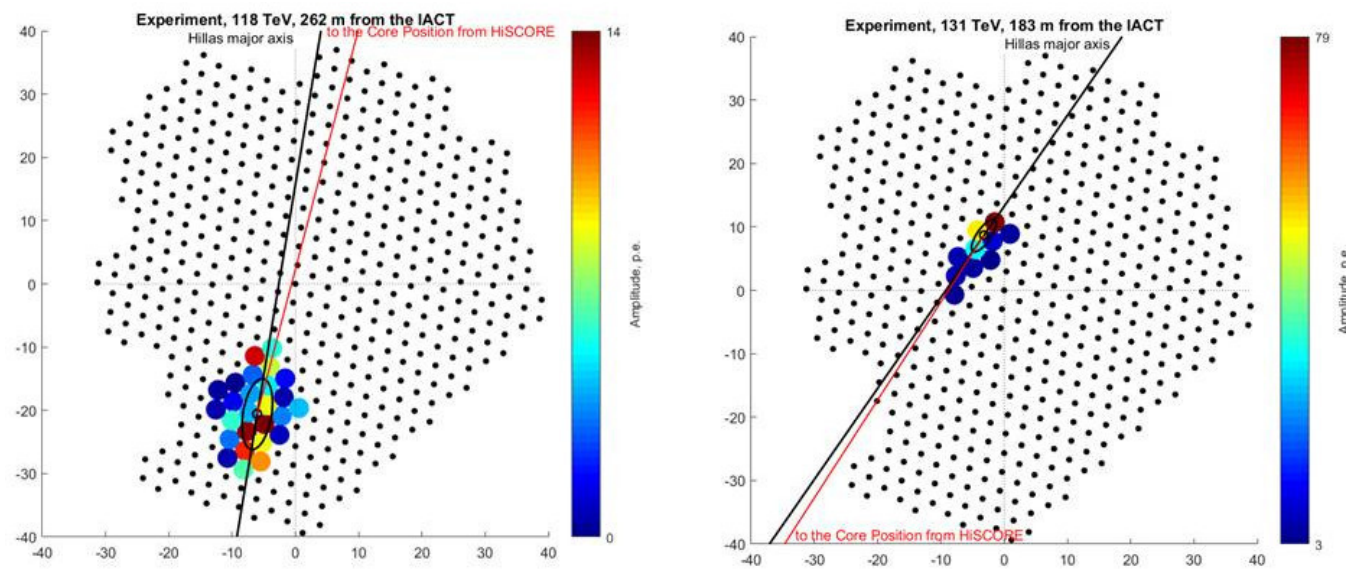

Figure 5: Examples of the images of joint events detected by the TAIGA-IACT and the TAIGA- HiSCORE. Red lines show the directions to the EAS core position, reconstructed by using the TAIGA- HiSCORE array data. Black lines - show the same and these are the major axes of the images.

For these events the shower core positions were determined using the TAIGA-HiSCORE data. The directions to these positions are shown by the red line on Fig.5. It is in good agreement with the major axis of the image obtained by the IACT (black line). Moreover, the measured image size distributions for all events and joint events are in good agreement with the results of the MC simulations [19]. The number of hit clusters of camera IACT in an event was within the limits 
from 1 to 19. In events with 10 or more hit clusters amplitudes of signals are nearly the same in all pixels. The counting rate of such events 10 hour $^{-1}$. As a rule, for such events there is a joint TAIGA-HiSCORE event. The estimated energy of these events are about $1 \mathrm{PeV}$ and the direction is outside the FOV of IACT. In our interpretation these events are from EAS Cherenkov light reflected from the snow around the IACT. Figure 5 shows two examples of the detected EAS images.

\section{The TAIGA-Muon array}

The total area of the muon detectors of the Tunka-Grande array [3] operated since 2015 is about $100 \mathrm{~m}^{2}$. With the help of this array it is possible to study the mass composition of CR above $10^{17} \mathrm{eV}$. For gamma-astronomy purposes the total area of muon detectors should be increased by 10 times at least. The cost of the installation is rather high due to the typical cost of a scintillation detectors of about 2000 EURO per square meter. We intend to develop efficient, low-cost, not served muon counters for the future TAIGA-Muon array, which are capable to operate for a long time under the two-meter layer of the wet soil.

The TAIGA-Muon counter have 4 scintillator sectors in the form of an isosceles triangle (Fig. 6). The length of sector hypotenuse is $100 \mathrm{~cm}$. Scintillator plates have crossection of $\sim 10 \times 160 \mathrm{~mm}^{2}$. Scintillator plates are located between wavelength shifting bars. It allows us to use PMTs with a small photocathode, and reduce the scintillator total volume. The cross section of the bar of the TAIGA-Muon counters is $5 \times 20 \mathrm{~mm}^{2}$. The light from the bars is detected by FEU-85 PMT (QE $=18 \%$ at $500 \mathrm{~nm}$ ). To have the same signal amplitude for different position of charged tracks we use one layer of scintillator in the central and middle parts of a sector and double layer of scintillator at periphery. The last results show that the full-scale counter can achieve the signal with the amplitude more than 23 photoelectrons. We assume that it will be enough to ensure $100 \%$ detection efficiency of a cosmic muon and a long lifetime of the detectors.
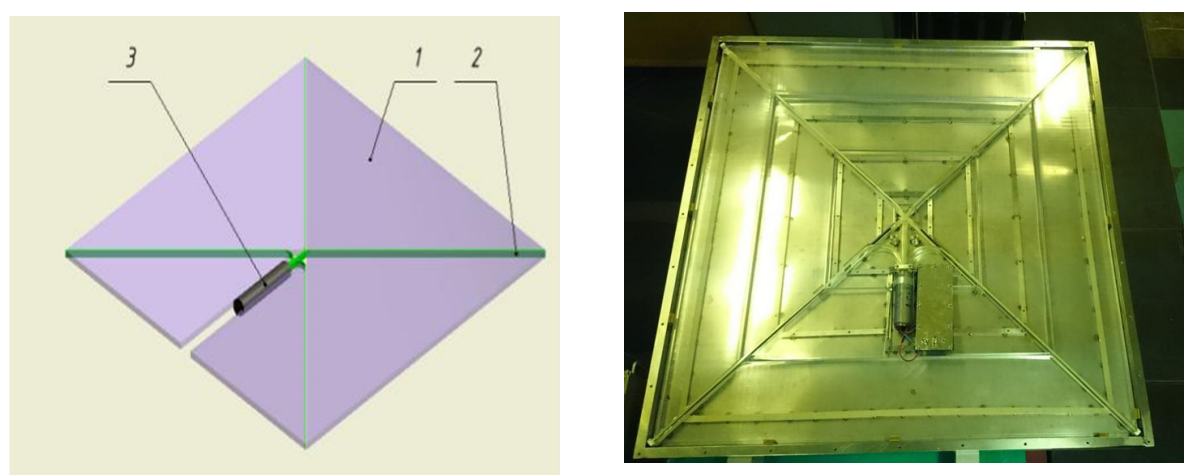

Figure 6: The TAIGA-Muon detector. 1-Scintillator, 2-Wavelength shifter, 3-PMT.

The first TAIGA-Muon counter with $1 \mathrm{~m}^{2}$ area will be deployed and tested at TAIGA site in July 2017. The first cluster with the 50 counters should be deployed in autumn 2017.

\section{Conclusion}

The expected integral sensitivity of the $1 \mathrm{~km}^{2}$ TAIGA setup for 300 hours source observation 
with at energy $100 \mathrm{TeV}$ will be about $2.5 * 10^{-13} \mathrm{TeV} \mathrm{cm}^{-2} \mathrm{sec}^{-1}$ (Fig.7) it will surpass the sensitivity of operated and planned installations in the ultra-high energy range. With the array it would be possible to solve follow objectives:

- Study of the high-energy edge of the spectrum of the most bright galactic gamma-ray sources.

- Search for Galactic Pevatrons.

- Apply the new hybrid approach (common operation of IACTs, wide-angle timing array and muon detectors) for study of cosmic rays mass composition in the "knee" region $\left(10^{14}-10^{16}\right.$ $\mathrm{eV})$.

- Explore the high energy region of the energy spectrum of the brightest extragalactic source Mkr421.

- Study of CR anisotropy in the energy region $100-3000 \mathrm{TeV}$

The TAIGA experiment will be the northernmost gamma-ray experiment, and its location provides advantages for observation of the sources with large declinations. So, gamma-ray source in the Tycho SNR, virtually inaccessible for HAWC [20] and LHAASO [21] will be in the field of view of the TAIGA during 500 hours per year.

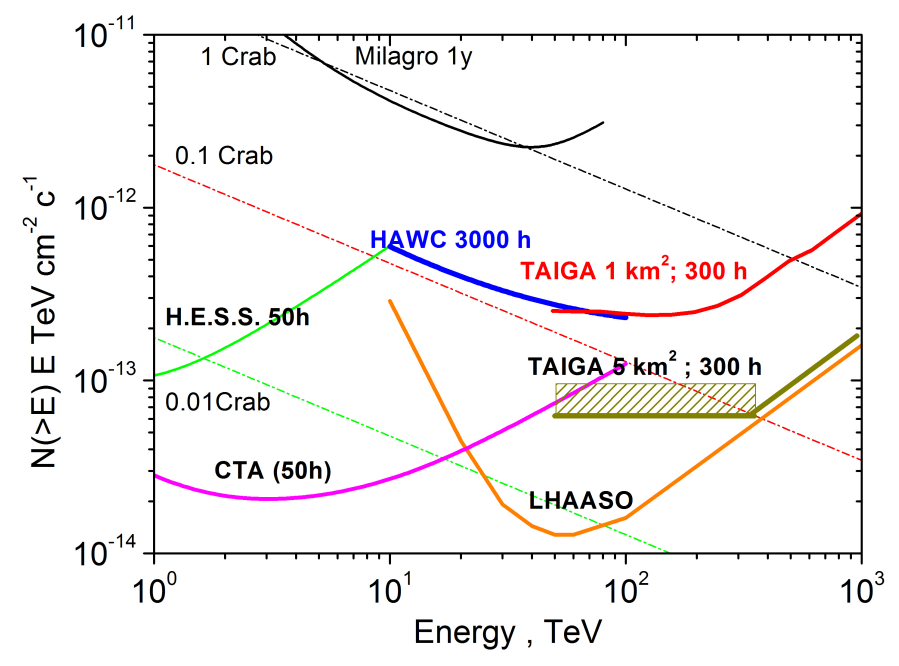

Figure 7: Integral sensitivity for detection of local sources.

\section{Acknowledgements}

This work was supported by the Russian Federation Ministry of Education and Science (projects 14.B25.31.0010, 2017-14-595-0001-003, No3.9678.2017/8.9, No3.904.2017/4.6, 3.6787.2017/7.8, 1.6790.2017/7.8), the Russian Foundation for Basic Research (Grants 16-02- 00738, 16-32-00329, 17-02-00905, 16-29-13035) and the grant 15-12-20022 of the Russian Science Foundation (section 2 and 3), by the Deutsche Forschungsgemeinschaft (DFG) and by the Helmholtz Association (HRJRG-303) and by European Union's Horizon 2020 programme (No.653477). 


\section{References}

[1] S. Berezhnev et al. (Tunka Collaboration), NIM A 692, 98 (2012)

[2] P.A. Bezyazeekov et al. (Tunka-REX Collaboration), JCAP 01, 052 (2016)

[3] R. Monhoev et al. (Tunka Collaboration), Bull. Russian Academy of Sciences: Physics, 81, 504 (2017)

[4] CTA website: https://www.cta-observatory.org/

[5] R. Mirzoyan, et al. (TAIGA collaboration), in 40th COSPAR Sc.Ass (2014), Vol. 40

[6] N. Budnev et al. (TAIGA Collaboration), NIM A 845, 384 (2017)

[7] M. Tluczykont et al., Astropaticle Phys. 56, 42 (2014)

[8] L.Kuzmichev et al (TAIGA Collaboration) EPJ Web of Conf. V 145 (2017) 01001

[9] S.F. Berezhnev et al. (TAIGA Collaboration), Bull. Russ. Acad. Sci. Phys.79 38 (2015)

[10] O.Gress et al. (TAIGA Collaboration), NIM A 845,367 (2017). A.Porelli et al (TAIGA-Coll.), J.Phys.:Conf.Ser. 632, 012041 (2015).

[11] V.Prosin et al (Tunka Collaboration), NIM A 756, 94 (2014)

[12] D. Hampf et al. NIM A 712 (2013) 137

[13] M. Tluczykont et al(TAIGA Collaboration) TAIGA-HiSCORE: results from the first two operation seasons, these proceedings. These conference proceedings

[14] http://cats.gsfc.nasa.gov/

[15] L.Sveshnikova et al (TAIGA Collaboration) The search for gamma emission above $50 \mathrm{TeV}$ from Crab Nebula in the TAIGA observatory. These conference proceedings

[16] A.Porelli e $t$ al (TAIGA Collaboration) TAIGA-HiSCORE detection of the CATS-Lidar on the ISS as fast moving point source. These conference proceedings

[17] N. Lubsandorzhiev et al ( TAIGA Collaboration) Camera of the first TAIGA-IACT: construction and calibration. These conference proceedings

[18] D. Zhurov et al ( TAIGA Collaboration) Software design for the TAIGA-IACT telescope pointing and control system. These conference proceedings

[19] L. Sveshnikova et al ( TAIGA Collaboration) Commissioning the joint operation of the wide angletiming HiSCORE Cherenkov array with the first IACT of the TAIGA experiment. These conference proceedings

[20] HAWC website: http://www.hawc-observatory.org/

[21] G.Di Sciascio (LHAASO Collaboration). Nucl.Part.Phys.Proc. 279-281 (2016) 166-173. 